\title{
采用重水为氞源的电化学氛化反应研究
}

\author{
廉 菲 徐 坤*
}

(北京工业大学生命科学与化学学院 北京 100124)

\section{Chemical-Reductant-Free Electrochemical Deuteration Reaction Using Deuterium Oxide}

\author{
Lian, Fei Xu, Kun* \\ (College of Life Science and Chemistry, Beijing University of Technology, Beijing 100124)
}

有机分子中的氢原子被気取代往往可以带来额外 的特性和功能. 例如 $\alpha$-氛代氨基酸作为一种高效的蛋白 质标记，已被应用于核磁共振(NMR)、氢氛交换质谱 (HDX-MS)、X 射线晶体学和拉曼散射显微学中. 此外, 氛代的氨基酸与天然氨基酸仅有微小的差异, 但可为某 些药物分子提供增强的代谢特性 ${ }^{[1]}$. 因此, 建立高效、便 捷的氛化反应方法学具有重要的研究意义. 近年来, 多 种新颖的氞化反应方法学被报道出来, 常采用的氞代试 剂有氞气、氛代二甲亚砜、氛代乙醇、氛代乙腈和氛代 苯等 ${ }^{[2]}$. 由于重水是最容易获得的氝源, 因此直接使用 重水进行氛化反应, 具有经济绿色的优势. 尽管一些反 应方法学能够实现有机分子与重水的直接氞化反应, 但 是需要使用外部还原剂作为电子给体, 例如 $\mathrm{Mg} 、 \mathrm{SmI}_{2}$, $\mathrm{Zn} 、 \mathrm{Mn}$ 或不锈钢 304 等 $(\text { Scheme 1, a })^{[3]}$. 此外, 由于水 是氢物种的稳定存储体, 如果要利用重水中的氛则需要 大量的能量输入. 因此, 该类反应需要一个高能量的还 原剂来促使反应的进行, 这将导致碱性副产物的生成和 较差的官能团容忍性. 鉴于上述局限性, 在无外加还原 试剂的条件下, 直接利用 $\mathrm{D}_{2} \mathrm{O}$ 作为氞源进行氞化反应是 一项具有重要意义, 但充满挑战性的工作.

有机电合成利用洁净的电子作为氧化还原试剂, 其 作为一种绿色高效的合成手段得到了有机科研工作者 的高度关注 ${ }^{[4]}$. 受到电化学还原反应的启发, 程旭课题 组 ${ }^{[5]}$ 发展了一种新型高效的电化学氛化反应方法学 (Scheme 1, b). 该反应以廉价的重水作为氛源, 在无需 外加还原试剂的条件下即可实现 $\alpha, \beta$-不饱和羰基化合物 的氛代反应, 且反应具有较好的反应收率、较高的氛代 率和较广的底物适用范围. (a) Reported protocols: high-energy-reductant driven deuteration

$$
\begin{aligned}
& \mathrm{X}^{\mathrm{Y} Y}+2 \mathrm{D}_{2} \mathrm{O} \longrightarrow \mathrm{D}^{-\mathrm{M}]} \mathrm{X}_{\mathrm{Y}^{-}}{ }^{-\mathrm{D}}+2[\mathrm{MOD}] \\
& \text { [M]: } \mathrm{Sml}_{2}, \mathrm{Mn}, \mathrm{Na}, \mathrm{Zn} \cdots
\end{aligned}
$$

(b) Electrochemical-oxygen-evolution-driven deuteration

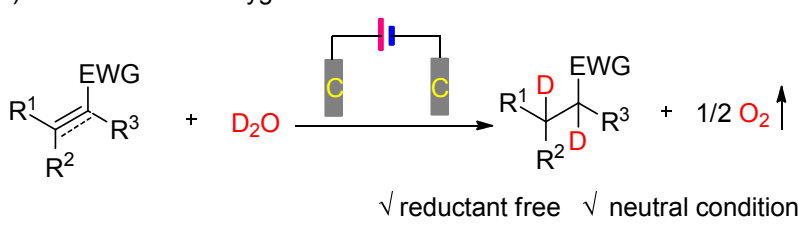

图式 1 以重水作为氞源的氛化反应

Scheme 1 Deuteration with $\mathrm{D}_{2} \mathrm{O}$ as the deuterium source

首先，作者选用末端带有环氧化物的 $\alpha, \beta$-不饱和羰 基化合物 $1 \mathrm{a}$ 作为模型底物, 和 $\mathrm{D}_{2} \mathrm{O}$ 进行氛化反应研究 (Scheme 2). 经过一系列反应条件优化，作者发现，当 使用 $n-\mathrm{Bu}_{4} \mathrm{NBF}_{4}$ 作为电解质, 石墨段(GF) 作为阳极和阴 极, $N, N$-二甲基甲酰胺(DMF)作为溶剂时, $1 \mathbf{a}$ 被気化为 饱和酯 2a 的产率最高可达到 $71 \%$, 且 $\alpha$ 和 $\beta$ 位置的氛

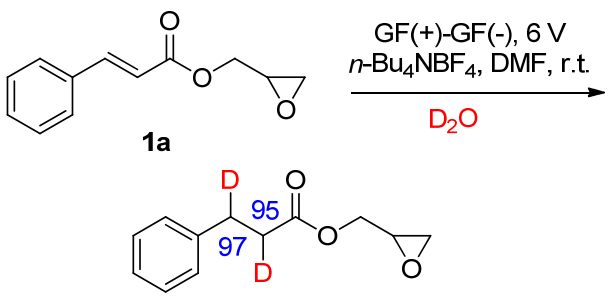

2a, $71 \%$ yield

图式 $2 \alpha, \beta$-不饱和羰基化合物的电化学氛代反应 Scheme 2 Electrochemical deuteration of $\alpha, \beta$-unsaturated carbonyl compounds using $\mathrm{D}_{2} \mathrm{O}$ as deuterium source

* Corresponding author. E-mail: kunxu@bjut.edu.cn. Published online September 3, 2020. 
代率分别为 $95 \%$ 和 $97 \%$. 如果采用 $\mathrm{Pt}$ 阴极, 则反应的转 化率和氝代率都会显著下降. 值得注意的是, 在反应过 程中环氧结构仍然完整保持.

进一步研究发现, 该电化学氝代反应方法学具有很 广的底物适用范围. 在最优的反应条件下, 多种烯烃和 炔烃化合物都能顺利地进行反应, 而且具有很高的気化 率(Scheme 3). 更为重要的是, 多种含有肉桂酸酯结<smiles>[R]C(=O)C([R])=C([R])[R]</smiles>

1
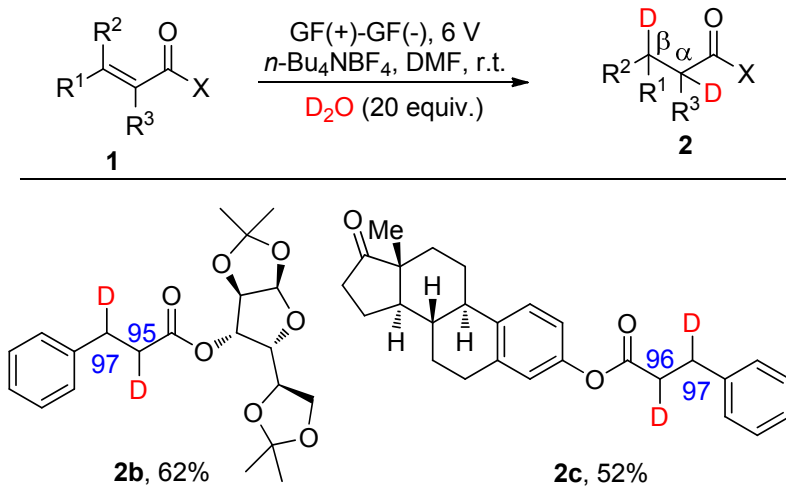

from diacetone- $D$-glucose

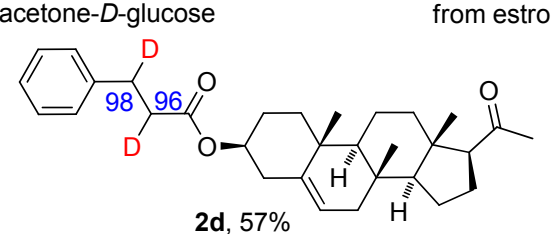

2d, $57 \%$

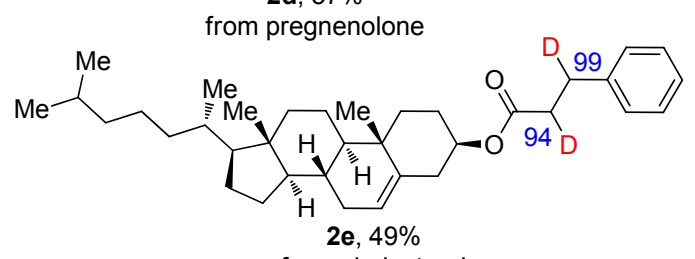

from cholesterol

图式 3 电化学氞化反应代表性底物

Scheme 3 Representative examples of electrochemical deuteration reaction
构的天然产物都可以在该条件下进行电化学気代反应. 此外，作者对気代的羧酸产物进行了一系列的衍生化合 成, 获取了多种氝代的官能化合物(例如嗍烷、炔烃、叠 氮化物、烷基苯并噻唑和烷基咖啡因等). 值得注意的 是, 在所有这些衍生化反应中, 没有观察到気的损失.

最后, 作者通过机理研究发现阳极析出的氧气对反 应起到至关重要的作用. 一方面, 氧气的析出可以避免 外加还原试剂的使用; 另一方面, 氧气的析出可以调控 反应的 $\mathrm{pH}$ 值，使其一直保持在中性范围.

综上, 程旭课题组创造性地发展了一种重水作为気 源的电化学氝化反应新方法, 反应具有较高的收率、氞 化率和较好的官能团容忍性. 由于该方法只需使用廉价 的石墨毡作为电极，且反应在室温下即可顺利进行，因 此和以往报道的还原剂介导的忥代反应相比, 该方法具 有经济绿色的显著优势. 此外，该反应可以放大到 $15 \mathrm{~g}$ 规模，一些重要的药物分子都可以利用该方法进行高效 率的氛代, 这进一步例证了该电化学氛化反应新方法的 实用性.

\section{References}

[1] Claydon, A. J.; Thom, M. D.; Hurst, J. L.; Beynon, R. J. Proteomics 2012, 12, 1194.

[2] Wang, Y.; Huang, Z.; Leng, X.; Zhu, H.; Liu, G.; Huang, Z. J. Am. Chem. Soc. 2018, 140, 4417.

[3] (a) Zhang, M.; Yuan, X.-A.; Zhu, C.; Xie, J. Angew. Chem., Int. Ed. 2019, 58,312 .

(b) Zhao, C.-Q.; Chen, Y.-G.; Qiu, H.; Wei, L.; Fang, P.; Mei, T.-S. Org. Lett. 2019, 21, 1412.

[4] (a) Jiang, Y.-Y.; Xu, K.; Zeng, C.-C. Chem. Rev. 2018, 118, 4485. (b) Sauer, G. S.; Lin, S. ACS Catal. 2018, 8, 5175.

[5] Liu, X.; Liu, R.; Qiu, J.; Cheng, X.; Li, G. Angew. Chem., Int. Ed. 2019, 59, 13962

(Zhao, C.) 